\title{
LINEAR PARTIAL DIFFERENTIAL EQUATIONS WITH CONSTANT COEFFICIENTS : AN ELEMENTARY PROOF OF AN EXISTENCE THEOREM
}

\author{
by D. H. PARSONS
}

1. We consider a linear partial differential equation with constant coefficients in one dependent and $m$ independent variables, the right-hand side being zero,

$$
\begin{gathered}
P\left(D_{1}, \ldots, D_{m}\right) z=0 \\
\left(D_{i} z \equiv \partial z / \partial x_{i}, i=1, \ldots, m\right),
\end{gathered}
$$

$P$ being a symbolic polynomial in $D_{1}, \ldots, D_{m}$. If $P$ can be decomposed into two factors, so that the equation can be written

$$
P\left(D_{1}, \ldots, D_{m}\right) z \equiv\left\{Q\left(D_{1}, \ldots, D_{m}\right) R\left(D_{1}, \ldots, D_{m}\right)\right\} z=0,
$$

it is evident that the sum of any solution of

$$
Q\left(D_{1}, \ldots, D_{m}\right) z=0
$$

and any solution of

is also a solution of ( 1 ).

$$
R\left(D_{1}, \ldots, D_{m}\right) z=0
$$

The converse proposition, that every integral of $(1)$ is the sum of an integral of (2) and an integral of (3), was postulated by Hadamard (1), in the case of $m=2$, for linear equations with constant or variable coefficients, provided only that the two operators $Q, R$ are commutative. This result was established by Cerf (2) and by Janet (3), who extended it to a very general case which certainly includes that under consideration here. The proof of the general theorem is not simple, however; and in the case mentioned below (§3), in which the equation is fully reducible, most textbooks are content to assume the result without proof.

We shall now give a purely elementary proof of this converse theorem, in the case when one of the factors of $P, R$ say, is a power of a linear expression in $D_{1}, \ldots, D_{m}$, which is not a factor of $Q$.

We shall make the hypothesis that any partial differential equation of the form

$$
T\left(D_{1}, \ldots, D_{m}\right) z=\phi\left(x_{1}, \ldots, x_{m}\right)
$$

admits at least one integral, provided only that $\phi$ satisfies sufficient conditions of continuity, and that the symbolic polynomial $T$ is not identically zero.

By suitable labelling, we may ensure that the linear factor of $P$ contains $D_{1}$. Thus let the equation considered be

$$
\left\{\left(D_{1}-a_{2} D_{2}-\ldots-a_{m} D_{m}-b\right)^{r} Q\left(D_{1}, \ldots, D_{m}\right)\right\} z=0,
$$

where $\left(D_{1}-a_{2} D_{2}-\ldots-a_{m} D_{m}-b\right)$ is not a factor of $Q$; and let us suppose that $z$ is an integral of this equation, admitting as many continuous partial derivatives as are required for the validity of the proof (or, if any of the 
constants $a_{2}, \ldots, a_{m}, b$ be complex, let $z$ be an analytic integral). We first substitute

$$
z=e^{b x_{1}} u ; x_{1}=y_{1} ; x_{i}+a_{i} x_{1}=y_{i} \quad(i=2, \ldots, m) .
$$

Then, taking account of the shifting formula, and writing $E_{j} u \equiv \partial u / \partial y$, $(j=1, \ldots, m)$, we see that $(4)$ becomes an equation of the form

$$
\left\{E_{1}^{r} T\left(E_{1}, \ldots, E_{m}\right)\right\} u \equiv E_{1}^{r}\left\{T\left(E_{1}, \ldots, E_{m}\right) u\right\}=0,
$$

where the symbolic polynomial $T$ is not divisible by $E_{1}$. Integrating $r$ times successively with respect to $y_{1}$, we see that $u$ satisfies the equation

$$
T\left(E_{1}, \ldots, E_{m}\right) u=\sum_{s=0}^{r-1}\left(y^{s} / s !\right) f_{s}\left(y_{2}, \ldots, y_{m}\right),
$$

for some form of the functions $f_{0}, f_{1}, \ldots, f_{r-1}$, which we again suppose admit enough continuous derivatives for our purpose.

The functions $f_{0}, \ldots, f_{r-1}$ being supposed known, the next step is to show that we can find a particular integral of the equation

$$
T\left(E_{1}, \ldots, E_{m}\right) V=\stackrel{r}{r=0}_{s}^{r-1}\left(y_{1}^{s} / s !\right) f_{s}\left(y_{2}, \ldots, y_{m}\right),
$$

which is of the same general form as the right-hand side. Let us rearrange $T\left(E_{1}, \ldots, E_{m}\right)$ in ascending powers of $E_{1}$. Then the equation to be satisfied by $V$ is ( $n$ being a positive integer or zero)

$$
\left\{\sum_{j=0}^{n} E_{1} S_{j}\left(E_{2}, \ldots, E_{m}\right)\right\} V=\stackrel{+}{i=0}_{s=0}^{+1}\left(y_{1}^{8} / s !\right) f_{s}\left(y_{2}, \ldots, y_{m}\right),
$$

where, since $T$ is not divisible by $E_{1}, S_{0}$ is not identically zero. Let

$$
V=\sum_{s=0}^{r-1}\left(y_{1}^{s} / s !\right) F_{s}\left(y_{2}, \ldots, y_{m}\right) \text {. }
$$

Then, substituting in (6), and equating coefficients of $y_{1}^{s}(s=0,1, \ldots, r-1)$ on the two sides, we see that we have an integral of (6) if $F_{0}, \ldots, F_{r-1}$ satisfy

$$
\left.\begin{array}{l}
S_{0}\left(E_{2}, \ldots, E_{m}\right) F_{r-1}=f_{r-1}\left(y_{2}, \ldots, y_{m}\right), \\
S_{0}\left(E_{2}, \ldots, E_{m}\right) F_{r-2}+S_{1}\left(E_{2}, \ldots, E_{m}\right) F_{r-1}=f_{r-2}\left(y_{2}, \ldots, y_{m}\right) \\
\vdots \\
\vdots \\
S_{0}\left(E_{2}, \ldots, E_{m}\right) F_{0}+\ldots=f_{0}\left(y_{2}, \ldots, y_{m}\right) .
\end{array}\right\}
$$

Since $S_{\mathbf{0}}$ is not identically zero, we can certainly find successively, in view of the hypothesis stated above, functions $F_{r-1}, F_{r-2}, \ldots, F_{0}$, which satisfy the equations (8), it being supposed that the $f_{i}$ admit enough continuous derivatives for this to be possible.

Suppose that $F_{r-1}, \ldots, F_{0}$ are a set of functions of $y_{2}, \ldots, y_{m}$ which have been chosen to satisfy (8), and let $V$ be the expression (7). Substituting $u=V+W$ in (5), and taking account of (7) and (8), we obtain

$$
T\left(E_{1}, \ldots, E_{m}\right) W=0 .
$$


Reverting to the variables $x_{1}, \ldots, x_{m}$, writing

$$
u=e^{-b x_{1} z}, \quad W=e^{-b x_{1} w},
$$

and taking account of the process by which $T\left(E_{1}, \ldots, E_{m}\right)$ was deduced from $Q\left(D_{1}, \ldots, D_{m}\right)$, we see that we have $z=v+w$, where

and $w$ satisfies

$$
v=e^{b x_{1}}{ }_{s=0}^{r-1}\left(x_{1}^{8} / s !\right) F_{s}\left(x_{2}+a_{2} x_{1}, \ldots, x_{m}+a_{m} x_{1}\right)
$$

$$
Q\left(D_{1}, \ldots, D_{m}\right) w=0
$$

It is easily verified directly that the expression $v$ above satisfies the equation

$$
\left(D_{1}-a_{2} D_{2}-\ldots-a_{m} D_{m}-b\right)^{r} v=0 \text { : }
$$

and we thus see that every integral of (4) is the sum of an integral of (10) and an integral of (11), the required result.

2. It is not possible to give minimum conditions of continuity for the validity of the above analysis; for, from the nature of the problem, these must vary from one example to another. For instance, if there be two dependent variables only, the general solution of

is clearly

$$
D_{1} z=0
$$

$$
z=f\left(x_{2}\right) \text {. }
$$

there being no restriction on the form of $f$. But the general solution of

$$
\left(D_{1}-D_{2}\right) z=0 \text { is } z=g\left(x_{1}+x_{2}\right) \text {, }
$$

and clearly $g$ must admit a derivative. The analysis is always valid, however, if we restrict our attention to analytic integrals of $(1)$.

3. In the case when the equation (1) is fully reducible, i.e., when $P\left(D_{1}, \ldots, D_{m}\right)$ can be expressed as a product of linear factors, it is clear that the sum of the various expressions such as (9), associated with each of the (repeated) factors of $P$, is an integral of (1) (4). Since this expression contains a number of arbitrary functions equal to the order of the equation, it is customary to assume that it is the general integral of (1). This assumption is now justified by repeated applications of the result obtained above.

\section{REFERENCES}

(1) Hadamard, Congrès de Zurich, 2 (1932), 80.

(2) Cerf, C.R. Acad. Sci., 197 (1933), 892.

(3) Janet, Ann. Soc. Polonaise de Math., 13 (1934), 85.

(4) I. N. Sneddon, Elements of Partial Differential Equations, ch. 3, §4 (McGrawHill, 1957).

\section{The University}

READING 